\title{
Exercising our options: comparing effects of exercise and positive expiratory pressure on mucociliary clearance
}

\author{
Scott H. Donaldson \\ Affiliation: Division of Pulmonary and Critical Care and Marsico Lung Institute, University of North Carolina at \\ Chapel Hill, Chapel Hill, NC, USA. \\ Correspondence: Scott H. Donaldson, Marsico Hall, CB\# 7248, University of North Carolina at Chapel Hill,
} Chapel Hill, NC 27599, USA. E-mail: scott_donaldsonamed.unc.edu

@ERSpublications

This editorial discusses the paper by Dwyer and co-workers: "Effects of exercise and airway clearance (PEP) on mucus clearance in cystic fibrosis", including implications for clinical care and future research http://ow.ly/3P6X30o6EaK

Cite this article as: Donaldson SH. Exercising our options: comparing effects of exercise and positive expiratory pressure on mucociliary clearance. Eur Respir J 2019; 53: 1900510 [https://doi.org/10.1183/ 13993003.00510-2019].

Cystic fibrosis (CF) lung disease is characterised by defective clearance of airway secretions that are hyperconcentrated, viscous, and serve as the nidus of chronic polymicrobial infection. The pathophysiological events that link CFTR channel dysfunction to mucus obstruction of airways are multifactorial, complex and an ongoing source of debate $[1,2]$. Despite the availability of multiple "symptomatic therapies" that target mucus retention (hypertonic saline, mannitol, dornase alfa and mucolytics), mechanical clearance of airway secretions has continued to be a cornerstone of treatment across patient populations [3]. Indeed, even with the emergence of highly effective CFTR modulators, which target the basic CF defect and improve mucociliary clearance [4], mechanical airway clearance techniques (ACT) are typically continued.

A large number of techniques and devices can be used to accomplish mechanical mucus clearance. The common denominator that links these therapies is that they are time-consuming (20-30 min per treatment, two or more times per day) and labour intensive when done properly. Generally, the goal of ACT treatments is to facilitate the transport of abnormal airway secretions from peripheral to large airways, where cough can then be used to expel them from the lung. A large number of breathing techniques (e.g. active cycle of breathing, autogenic drainage and forced expiratory technique) and airway clearance devices (positive expiratory pressure (PEP) devices, oscillatory PEP, and high frequency chest wall oscillation vests) are available. Given the paucity of high quality research that is available to critically evaluate individual ACT methods, it is understandable their usage is highly variable across centres, and a significant amount of effort is expended to select a technique that is most efficacious and acceptable to an individual patient.

Exercise has long been recommended to patients with CF as an adjunctive ACT [5-7]. Regular aerobic exercise has been shown to improve exercise capacity, quality of life/symptoms, and to slow loss of lung function over time $[8,9]$. It is unclear, however, whether exercise can fully substitute for mechanical ACT, and thus provide adequate airway clearance while bringing additional health benefits that result from exercise training. 
Prior studies that attempted to assess or compare various modes of ACT and exercise have typically been short in duration and relied upon measurements of sputum weight, symptoms and/or sputum biomarkers of unclear clinical significance. Very few long term longitudinal studies using clinically meaningful outcomes (e.g. lung function, pulmonary exacerbation frequency) have been performed. Given the difficulty and cost of performing such trials, use of robust, though incompletely validated, outcome measures that assess integrated lung physiological responses, including mucociliary clearance, are attractive on account of their biological relevance.

In this issue of the European Respiratory Journal, DwYER et al. [10] performed a randomised, crossover trial to compare the physiological effect of a single exercise session on mucociliary clearance to that of PEP therapy, which included huffing and coughing manoeuvres, and a no intervention control session. 14 stable adults with a wide range of disease severity were enrolled. The exercise and PEP interventions were carefully standardised and controlled. Routine inhaled therapies (mucolytics, bronchodilators), exercise and usual airway clearance manoeuvres were held on study days until after research procedures were completed. Mucociliary clearance was measured using gamma scintigraphy, with careful control of radiolabelled particle inhalation parameters. Close attention was paid to the potential impact of the initial particle deposition pattern and cough frequency on clearance rates. Clearance rates immediately prior to intervention, during intervention and post-intervention were measured in the whole lung, as well as in lung subdivisions (central, intermediate and peripheral lung). Patient-reported "chest congestion" was also assessed with a visual analogue scale.

The investigators observed that treadmill exercise improved whole lung mucociliary clearance from baseline, but was less effective than PEP. Interestingly, when examining the regional effects of these interventions on mucociliary clearance, the difference between exercise and PEP resulted almost entirely from a large difference in central lung clearance (improved with PEP; no effect of exercise). PEP with cough improved clearance from all lung zones, whereas exercise improved it only in the intermediate and peripheral zones. Importantly, while subjects were observed to have a modest amount of involuntary cough during exercise (versus control study day), cough counts were far less than during PEP, which included voluntary cough manoeuvres as a part of the intervention. It seems likely, therefore, that this difference in cough explains the difference in central lung clearance. Interestingly, the relative impact of interventions on patient-reported chest congestion largely mirrored the differences in central lung clearance, i.e. strongly favoured PEP.

What can be learned from this study, and what additional work is required? First, this physiologically anchored study clearly differentiates the effects of exercise and a standard ACT on mucociliary clearance, providing data that can be used to guide clinical decisions. Further, by clarifying where mucociliary clearance is impacted by exercise, the option of adding huff/cough manoeuvres after exercise to increase large airway clearance emerges as a rational option. These data also contribute to our understanding of the mechanisms underlying effective mucus clearance in the lung. In $\mathrm{CF}$, as mucus properties become increasingly abnormal, with progressive dehydration and hyperviscosity, cilia-driven clearance begins to fail and cough becomes increasingly important, but is only effective in large airways where sufficient flow rates can be achieved [11]. In smaller airways, mucociliary clearance relies on cilia motion and, possibly, expiratory airflow to drive the proximal movement of secretions. Exercise is speculated to improve small airway clearance by stimulating release of mediators (e.g. adenosine triphosphate) that increase fluid secretion and stimulate cilia beat frequency [12]. One might also envision that the ventilatory changes that occur during exercise could also directly drive small airway mucus transport. Clearly, though, exercise appears to be ineffective by itself for central/large airway clearance. Further, the data from DwYER et al. [10] suggest that whatever the mechanisms are that underlie enhanced mucociliary clearance during PEP and exercise, their effects have a short duration, as no sustained improvement in clearance persisted once the intervention stopped. Therefore, wholesale exchange of exercise for ACT would probably not be advisable, given the short-duration effect of treatments and negligible likelihood of exercising multiple times a day.

Unfortunately, we do not yet know whether observed MCC effects can accurately predict and/or differentiate the clinical benefits that result from exercise and ACT of any type. Clearly, this limits our ability to confidently apply these data to clinical practice. However, given the difficulty of performing carefully controlled clinical trials of ACT and exercise interventions, having a reliable, physiologically relevant surrogate (e.g. mucociliary clearance, MRI-based ventilation imaging; multiple breath washout techniques) for these and other therapies that stimulate mucus clearance would be incredibly valuable. If predictive, such a tool would drive us to not only compare ACTs and exercise, but to address whether these and other mucolytic and mucokinetic therapies are useful after institution of the highly effective CFTR modulator treatments that are expected to play a central role in care for the majority of CF patients in coming years. In support of this idea, a recent publication did suggest that individual patient changes in 
mucociliary clearance in part predicted improvements in lung function (e.g. forced expiratory volume in $1 \mathrm{~s}$ ) following treatment with ivacaftor in G551D-CFTR patients [13]. Conceptually, one could imagine that assessments of lung ventilation, either via imaging or multiple breath washout techniques [14], could also be used to explore these questions.

Going forward, the study performed by DwYER et al. [10] helps us to understand the place of exercise in the care of CF lung disease, although this topic will require additional attention. The incorporation of relevant, physiological end-points into other clinical trials may guide us toward treatment approaches that are rational and most likely to meet the needs of individual patients. However, results of short-term trials will continue to require confirmation with longer studies that assess clinical outcomes until validated surrogates are available.

Conflict of interest: None declared.

\section{References}

1 Hill DB, Long RF, Kissner WJ, et al. Pathological mucus and impaired mucus clearance in cystic fibrosis patients result from increased concentration, not altered pH. Eur Respir J 2018; 52: 1801297.

2 Tang XX, Ostedgaard LS, Hoegger MJ, et al. Acidic $\mathrm{pH}$ increases airway surface liquid viscosity in cystic fibrosis. J Clin Invest 2016; 126: 879-891.

3 Flume PA, Robinson KA, O'Sullivan BP, et al. Cystic fibrosis pulmonary guidelines: airway clearance therapies. Respir Care 2009; 54: 522-537.

4 Rowe SM, Heltshe SL, Gonska T, et al. Clinical mechanism of the cystic fibrosis transmembrane conductance regulator potentiator ivacaftor in G551D-mediated cystic fibrosis. Am J Respir Crit Care Med 2014; 190: 175-184.

5 Baldwin DR, Hill AL, Peckham DG, et al. Effect of addition of exercise to chest physiotherapy on sputum expectoration and lung function in adults with cystic fibrosis. Respir Med 1994; 88: 49-53.

6 Zach M, Oberwaldner B, Hausler F. Cystic fibrosis: physical exercise versus chest physiotherapy. Arch Dis Child 1982; 57: 587-589.

7 Ward N, Stiller K, Holland AE. Exercise is commonly used as a substitute for traditional airway clearance techniques by adults with cystic fibrosis in Australia: a survey. J Physiother 2019; 65: 43-50.

8 Schneiderman-Walker J, Pollock SL, Corey M, et al. A randomized controlled trial of a 3-year home exercise program in cystic fibrosis. J Pediatr 2000; 136: 304-310.

9 Joschtel B, Gomersall SR, Tweedy S, et al. Effects of exercise training on physical and psychosocial health in children with chronic respiratory disease: a systematic review and meta-analysis. BMJ Open Sport Exerc Med 2018; 4: e000409.

10 Dwyer TJ, Daviskas E, Zainuldin R, et al. Effects of exercise and airway clearance (positive expiratory pressure) on mucus clearance in cystic fibrosis: a randomised crossover trial. Eur Respir J 2019; 53: 1801793.

11 Pavia D, Agnew JE, Clarke SW. Cough and mucociliary clearance. Bull Eur Physiopathol Respir 1987; 23: Suppl. $10,41 \mathrm{~s}-45 \mathrm{~s}$.

12 Button B, Okada SF, Frederick CB, et al. Mechanosensitive ATP release maintains proper mucus hydration of airways. Sci Signal 2013; 6: ra46.

13 Donaldson SH, Laube BL, Corcoran TE, et al. Effect of ivacaftor on mucociliary clearance and clinical outcomes in cystic fibrosis patients with G551D-CFTR. JCI Insight 2018; 3: 122695.

14 Smith LJ, Collier GJ, Marshall H, et al. Patterns of regional lung physiology in cystic fibrosis using ventilation magnetic resonance imaging and multiple-breath washout. Eur Respir J 2018; 52: 1800821. 